\title{
Retour d'expérience sur la régulation au Samu de Paris pendant la crise de Covid-19
}

\author{
Feedback on the Regulation of Samu de Paris during the COVID-19 Crisis
}

\author{
C. Telion $\cdot$ J.-S. Marx $\cdot$ C. Dautreppe $\cdot$ P. Carli
}

Reçu le 22 juillet 2020 ; accepté le 8 septembre 2020

(C) SFMU et Lavoisier SAS 2020

Résumé L'épidémie de Covid-19 représente une crise dont l'ampleur n'avait jusque-là jamais été imaginée. Des modifications des pratiques pour y faire face ne pouvaient reposer uniquement sur des doctrines ou des intuitions, mais nécessitaient adaptabilité, innovation et réactivité. Un renforcement en moyens techniques et humains a été débuté dès la fin du mois de février. L'organisation de la régulation médicale du Samu de Paris a été modifiée, renforcée en fonction des flux de patients à traiter et adaptée à la spécificité de la crise de Covid. L'ensemble des mesures avait comme objectif d'apporter la réponse la plus adaptée aux patients atteints de la Covid-19 et de préserver la réponse aux appels urgents du 15. Une collaboration fructueuse s'est rapidement établie entre tous les acteurs de la santé, hospitaliers et libéraux favorisant un maintien à domicile d'un certain nombre de patients et empêchant ainsi une saturation précoce des services d'urgence. Le développement et l'intégration de nouveaux outils informatiques ont facilité et diversifié les réponses apportées. Il est, dès à présent, indispensable de pérenniser et de renforcer ces acquis afin de développer le service d'accès aux soins (SAS) nécessaire pour apporter à la population une qualité de soins optimisée.

Mots clés Covid-19 $\cdot$ SARS-Cov-2 $\cdot$ Samu $\cdot$ Régulation médicale $\cdot$ Situation sanitaire exceptionnelle

Abstract COVID-19 represents a crisis the scale of which
had never been imagined before. Changes in practices to
coping with them could not be based solely on doctrines

C. Telion $(\bowtie) \cdot$ J.-S. Marx $\cdot$ C. Dautreppe $\cdot$ P. Carli Samu de Paris, hôpital Necker-Enfants-Malades, Assistance publique-Hôpitaux de Paris, F-75015 Paris, France e-mail : caroline.telion@aphp.fr

P. Carli

Université de Paris, F-75270 Paris, France or intuitions, but require adaptability, responsiveness, and innovation. A reinforcement of technical and human resources was introduced at the end of February. The organization of the medical regulation of the Samu (Emergency Medical Service) in Paris has been modified, reinforced according to the flow of patients to be treated and adapted to the Covid evolution. All the measures were aimed at providing the most appropriate response to patients with COVID-19 and preserving the answer of the emergency calls received on the 15. A fruitful collaboration was quickly established between all the actors of health, particularly with in-hospital and liberal practitioners favoring a home maintenance of a lot of patients and thus avoiding the saturation of emergency medical services. The development and integration of new IT tools have facilitated and diversified the provided solutions. It is now essential to perpetuate and strengthen these achievements to develop the service to access to care (French acronym SAS).

Keywords COVID-19 $\cdot$ SARS-CoV-2 $\cdot$ SAMU $\cdot$

Emergency medical service $\cdot$ Medical regulation $\cdot$ Call center $\cdot$ Health crisis

\section{Introduction}

Le 5 janvier 2020, la Chine révèle l'émergence d'un nouveau coronavirus, dont la gravité, le mode de contamination ne sont pas déterminés, sur son territoire dans la ville de Wuhan province du Hubei. Le 21 janvier, le ministère de la Santé lance un communiqué incitant «toute personne ayant voyagé en Chine, principalement dans la province du Hubei, et présentant de la fièvre, à "ne pas se rendre chez son médecin traitant ni dans un service d'urgence, mais à appeler le 15" ". Dès lors, les services d'aide médicale d'urgence (Samu)-Centre de réception et de régulation des appels (CRRA) 15 se trouvent en première ligne de ce qui va devenir la plus grande crise 
sanitaire depuis la Seconde Guerre mondiale. La mise en place du plan ORSAN REB [1] et la diffusion le 20 février du guide méthodologique "Préparation au risque épidémique Covid-19 " [2] conduisent chaque SamuCentre 15 à anticiper un renforcement de sa régulation du fait des possibilités d'augmentation du nombre d'appels. En région Île-de-France, le Samu de Paris est l'un des quatre Samu dépendant de l'Assistance publique-Hôpitaux de Paris (AP-HP). Il est identifié comme le Samu zonal et doit en cas de crise coordonner l'action interdépartementale des huit Samu de la région Île-de-France. L'ensemble des mesures, qui sont rapportées plus loin, ont été mis en place au Samu de Paris. Ces mesures sont le fruit d'un partage des connaissances et de l'expérience acquises en temps réel entre les huit Samu d'Île-de-France, notamment au cours d'une conférence téléphonique zonale quotidienne. Ces mesures découlent de l'application du plan ORSAN REB et du pilotage national de la crise transmis aux professionnels de santé par le Centre de régulation et de réponse aux urgences sanitaires et sociales (CORRUSS) du ministère de la Santé.

\section{Chronologie de l'organisation}

L'activité de la régulation médicale, au cours de la crise, peut schématiquement être divisée en trois périodes successives dont les effets sur le fonctionnement se sont additionnés et complétés sur une durée de plus de trois mois (Fig. 1).

De fin janvier à fin février, la régulation médicale a participé très activement à la recherche des premiers cas, importés ou nationaux, en lien direct avec les services hospitaliers de référence, en déterminant suivant le dispositif national, les cas possibles, les cas probables et les contacts suspects. La régulation médicale a permis l'hospitalisation des cas avérés en services spécialisés de maladies infectieuses et l'orientation des autres cas vers la médecine de premier recours. Ainsi, de nombreux patients peu gravement atteints ont bénéficié d'une orientation par les médecins régulateurs de la permanence des soins (PDS) vers les médecins généralistes, les cas plus graves étaient pris en charge par la régulation de l'aide médicale d'urgence (AMU). Pendant cette période, le gouvernement français a proposé à ses ressortissants habitants la région de Wuhan, qui le souhaitaient, un

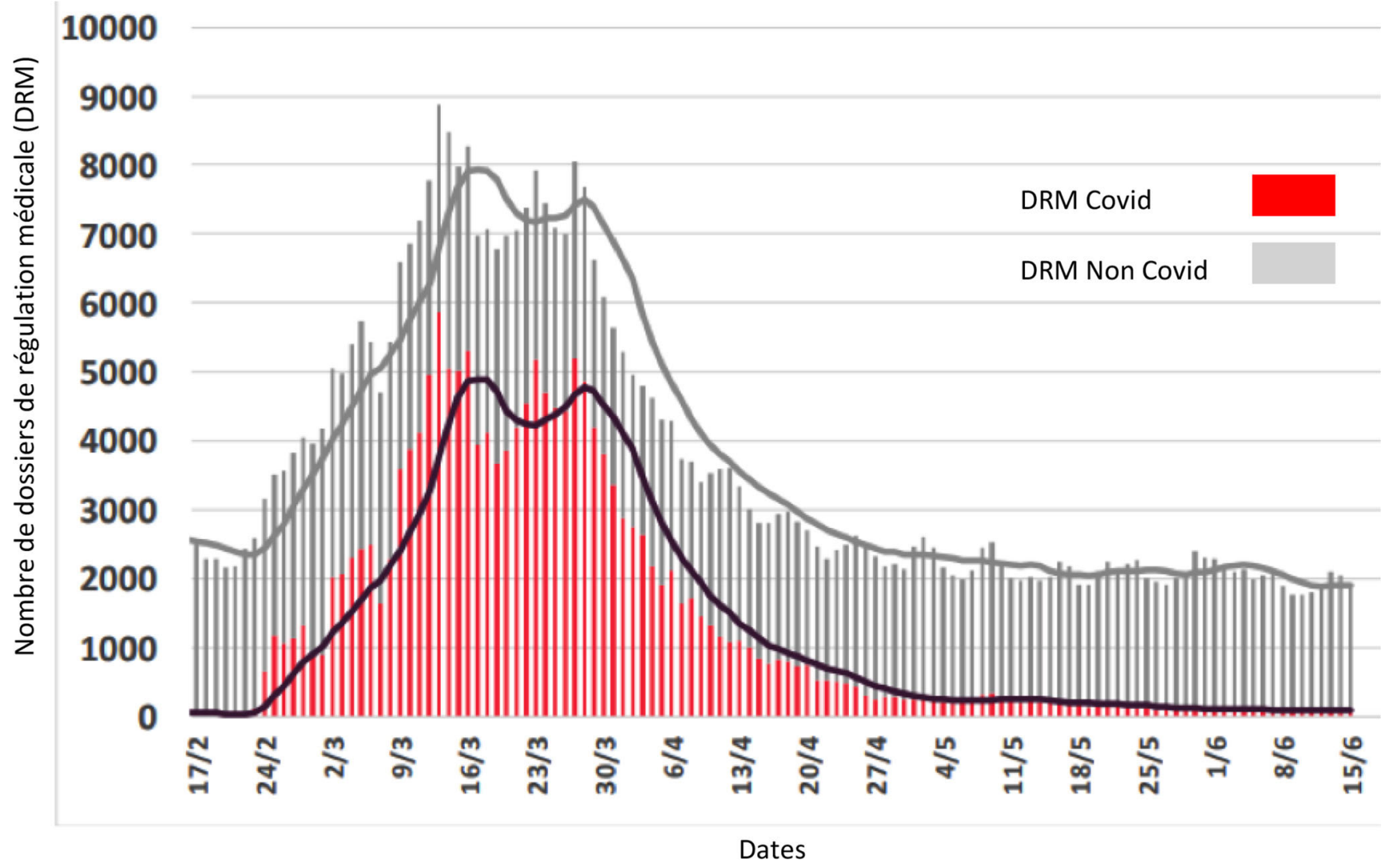

Fig. 1 Évolution du nombre de dossiers de régulation médicale (DRM) ouverts par jour dont les DRM Covid-19 par les quatre Samu de l'Assistance publique-Hôpitaux de Paris (AP-HP) figurant dans le tableau de bord quotidien de la cellule de crise AP-HP. Les courbes correspondent aux moyennes mobiles sur sept jours 
rapatriement sur le territoire national. Ainsi, trois vols ont été affrétés les 28 et 31 janvier puis le 19 février, la médicalisation a été assurée par des personnels de différents Smur d'Île-de-France, le Samu de Paris en a assuré la coordination au titre de sa fonction zonale. Cette première étape a mis en évidence la spécificité de la typologie des appels Covid-19 et la possibilité d'organiser la régulation médicale en identifiant ces appels et en mettant en place une filière de réponse dédiée qui sera optimisée tout au long de la crise.

La deuxième phase débute à la fin du mois de février (vers le 24 février), les appels au 15 concernant le Covid augmentent sensiblement pour atteindre un pic historique le 13 mars avec 26000 appels pour les quatre Samu AP-HP en 24 heures, dont 8400 à Paris, soit plus de quatre fois l'activité maximale jusque-là observée. L'adaptation de la régulation médicale a eu alors deux objectifs principaux :

- améliorer la réponse au flux d'appels en préservant les appels d'urgence du 15 ;

- répondre efficacement et spécifiquement aux appels Covid-19 pour éviter les passages inutiles aux services d'accueil des urgences (SAU) qui risqueraient alors d'être rapidement saturés.

Devant cet afflux d'appels exceptionnel et imprévisible, des mesures (dont certaines étaient en préparation depuis quelques jours) ont été déployées immédiatement. Ce pic d'appels n'a pas entraîné d'augmentation majeure des transports vers l'hôpital, car une majorité de patients a été pris en charge en ambulatoire et cela a permis d'améliorer rapidement la réponse aux appels et de faire face efficacement à un deuxième pic atteint le 24 mars, en revenant à des performances comparables à celles d'avant la crise.

Avec ce deuxième pic d'appels débute la troisième phase. En effet, ce pic est alors suivi de nombreuses admissions aux SAU et directement en réanimation témoignant de la gravité des patients appelants le 15 . Ainsi, à partir de fin mars et de début avril, à l'activité précédente s'ajoutent une multiplication des transports Smur, des interventions non médicalisées à domicile et des transports sanitaires nécessitant des recherches de place sur la région Île-de-France. Cette activité a aussi imposé de nombreux transports interhospitaliers pour exploiter au mieux les capacités de réanimation existantes ou créées de novo dans les établissements hospitaliers, publics comme privés. C'est dans ce contexte que la régulation du Samu de Paris a participé au titre de Samu zonal à la demande du CORRUSS, à l'organisation des transports interhospitaliers par vecteurs médicalisés terrestres, aériens, ou ferroviaires au départ de l'Île-de-France vers les réanimations des zones de défense Ouest et Sud-Ouest [3].

Enfin, au milieu du mois d'avril, l'activité de la régulation médicale a décru lentement jusqu'au milieu du mois de mai, alors que dans les services de réanimation l'activité restait très intense.

\section{Adaptation de l'organisation de la régulation médicale}

Pour répondre quantitativement et qualitativement à la crise de Covid-19, les équipes du Samu de Paris ont dû faire preuve d'innovation, d'adaptabilité et de réactivité. Des mesures ont été déployées non seulement conformément aux plans et instructions existants, mais aussi dans un but pragmatique d'atteindre rapidement le résultat demandé. Les principales modifications de la régulation médicale portent sur trois domaines : la technologie, l'organisation de la régulation et les personnels.

Une équipe d'ingénieurs et de techniciens de la direction du service informatique de l'AP-HP (DSI-AP-HP) a été déployée $24 \mathrm{~h} / 24$ dans les quatre Samu AP-HP. Elle était associée au sein du Samu de Paris à une équipe de chercheurs de l'École polytechnique-INRIA, facilitant la mise en œuvre des solutions retenues, les analysant en temps réel afin de les adapter le plus rapidement possible [4]. L'ensemble des mesures réalisées ont été partagées en temps réel entre les quatre Samu AP-HP, ainsi qu'avec les quatre autres Samu de la région Île-de-France et comparé avec les solutions adoptées, notamment dans la région Grand Est, mais aussi dans les diverses capitales européennes (dans le cadre du réseau EMS leadership Network) et nord-américaines (au cours des conférences du EAGLES Network ${ }^{1}$.

\section{Améliorations techniques}

Le raccordement au réseau téléphonique de France Télécom du Samu est établi grâce à un accès primaire appelé T2. Pour répondre aux appels, il a été nécessaire, à Paris, de multiplier par quatre les T2 permettant de passer de 40 appels simultanés à 120 le 13 mars puis à 180 le 24 mars. Dès leur ouverture, ces lignes ont fonctionné à plein régime. Pour faire face à la vague d'appels entrants par les canaux ainsi ouverts, tous les postes de régulation médicale existants ont été activés et dotés en personnels. Vingt postes informatiques supplémentaires ont été créés, soit une multiplication par 2,4 des postes, avec 45 postes actifs et l'ouverture de plus de 200 licences d'utilisation. Les postes de régulation médicale ont été répartis dans trois salles de régulation supplémentaires dans les locaux du Samu de Paris, permettant l'utilisation de quatre salles ouvertes $24 \mathrm{~h} / 24$, dont les configurations ont été adaptées à l'activité et à la nature des appels.

\footnotetext{
${ }^{1}$ The EAGLES est une association, créée en 1998 par le Pr Paul Pepe, qui regroupe des directeurs et des médecins responsables de services d'urgences préhospitaliers des grandes villes nord- américaines et de quelques homologues de grandes villes du monde comme Paris, Berlin, Auckland.
} 


\section{Modifications de l'organisation de la régulation}

Les modifications de l'organisation de la régulation médicale du Samu de Paris ont été précoces, multipliées et renforcées en fonction des analyses des temps de décroché et des flux de patients à traiter.

Dès le début du mois de février, les différents messages diffusés dans les médias sur la Covid-19 ont généré des doutes, des inquiétudes dans la population qui a appelé le 15 pour bénéficier de conseils, d'explications par rapport à des messages parfois anxiogènes ou contradictoires. Les assistants de régulation médicale (ARM) ont donc transformé leur interrogatoire en demandant d'emblée si l'appel concernait un conseil sur la Covid-19, ne prenant plus l'adresse du patient, transmettant immédiatement l'appel à un médecin afin de libérer la ligne le plus rapidement possible. Après avoir analysé l'appel, si le médecin décidait l'envoi d'un moyen, il remplissait lui-même les coordonnées du patient et transmettait cette demande de moyens par voie informatique à un personnel dédié.

La mise en place d'une surveillance quotidienne du nombre d'appels Covid par un codage spécifique réalisé par les ARM, dès le décroché du 15 , a permis l'activation progres- sive des demandes de moyens techniques et humains. À la fin du mois de mars, le dispositif mis en place a atteint son maximum et permis de prendre en charge le plateau d'activité très intense qui a suivi le premier pic d'appel du 13 mars. La figure 2 résume le traitement de l'appel.

Les mesures suivantes ont été successivement ou simultanément effectives :

- mise en place d'un serveur vocal interactif(SVI) orientant les appels Covid vers une filière de réponse spécifique, préservant ainsi la réponse aux appels 15 classiques qui restaient pris en charge par les personnels Samu. Ce dispositif opérationnel $24 \mathrm{~h} / 24$ dès la seconde quinzaine de mars a permis d'orienter, au moment des pics d'appels, plus de $90 \%$ des appels vers la filière Covid;

- mise en place d'un niveau N1 de décroché très rapide dans la filière Covid. Un tri initial des appels Covid a été mis en place à partir du décroché de l'ARM pour libérer le plus rapidement possible les ARM les plus expérimentés. À partir de ce niveau N1, si l'ARM détectait un signe de gravité, il passait immédiatement l'appel au niveau 2 Covid AMU, c'est-à-dire au médecin régulateur AMU. Si l'ARM Covid N1 ne détectait aucun signe de gravité, il transmettait l'appel au niveau 2 Covid non AMU, en salle de crise Covid où un étudiant

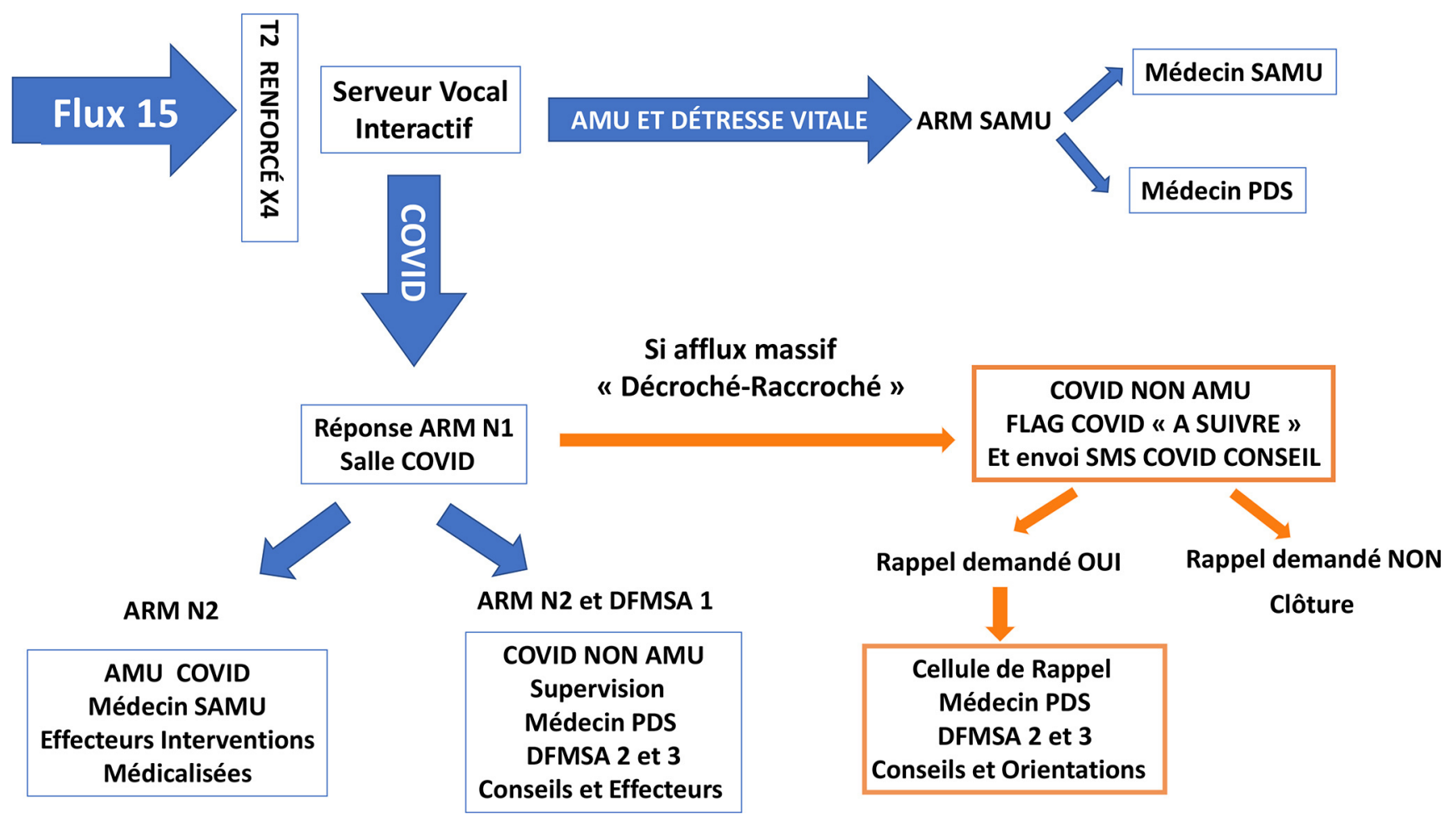

Fig. 2 Gestion des flux d'appels en régulation après le 14 mars 2020 au Samu de Paris. AMU : aide médicale urgente, PDS : permanence des soins, ARM : assistant de régulation médicale, DFMSA : diplôme de formation médicale spécialisée approfondie, N1 : premier niveau de réponse au décroché du 15 déterminant la gravité de l'appel et son orientation, N2 : deuxième niveau de réponse : précision et traitement de la demande 
en médecine de diplôme de formation approfondie en sciences médicales (DFASM) de $1^{\text {re }}$ année prenait l'appel, notait les coordonnées du patient et le transmettait à un autre étudiant de DFASM de $2^{\mathrm{e}}$ et $3^{\mathrm{e}}$ années. Ce dernier, en l'absence de symptomatologie, répondait aux questions du patient, ou en cas de symptomatologie minime proposait au patient de rentrer dans une structure de suivi à domicile (Covidom) de l'évolution de la symptomatologie et de dépistage précoce de toute aggravation [5]. Les 20 postes informatiques de régulation médicale supplémentaires ont été dédiés exclusivement aux patients appelant pour une pathologie Covid peu grave compatible avec un traitement ambulatoire ou un conseil médical. Dans les deux salles de régulation supplémentaires où ils ont été déployés, l'activité des jeunes médecins était encadrée par un médecin référent senior de la PDS et/ou de l'AMU. Ainsi, tout patient présentant des signes de gravité potentiels dépistés lors de la prise de l'appel ou au cours d'un interrogatoire approfondi était redirigé vers la régulation de l'AMU ;

- mise en place d'une stratégie de « décroché-raccroché ». Ce procédé avait pour but de raccrocher pour libérer rapidement les lignes téléphoniques entrantes pour traiter de nouveaux appels, et d'utiliser d'autres lignes pour rappeler et répondre à la demande non urgente d'un patient. Ainsi, toute demande de conseil concernant la Covid conduisait à l'ouverture d'un dossier de régulation médicale avec la notification uniquement du numéro d'appel et de l'arrondissement de Paris et un marquage spécifique avec un «flag ». L'appel était ensuite raccroché par l'ARM avec l'assurance auprès de l'appelant d'un rappel par un membre de l'équipe médicale. Ce rappel, non urgent, était assuré dès que possible par des étudiants de DFASM de $2^{\mathrm{e}}$ et $3^{\mathrm{e}}$ années ou par les transmetteurs (association de médecins volontaires retraités) renforçant le Samu. La technique du "décroché-raccroché »a été déployée dans la phase d'activité la plus intense, notamment de jour et en début de soirée. Cette méthode de réponse différée a été appréciée par les appelants qui ont manifesté leur satisfaction d'être rappelé et de disposer alors du temps nécessaire de dialogue avec un médecin pour exposer leur cas. Le "décroché-raccroché » a été complété par un système d'information (SI) rapide par SMS qui a permis de diminuer sensiblement la nécessité des rappels de patients. En effet, un grand nombre d'appels étaient liés à l'inquiétude que pouvait ressentir la population parisienne face à l'ensemble des informations diffusées sur les médias. Pour répondre aux appelants qui ne présentaient aucun symptôme et souhaitaient une réassurance, à partir du 12 mars un message (SMS) donnant des explications très didactiques sur la Covid-19 a été automatiquement associé à chaque appel décroché-raccroché. Ce SMS offrait la possibilité d'être rappelé ou non par le Samu en fonction de la satisfaction apportée par l'information reçue. Deux mille cinq cent trois SMS ont été dispensés, le taux de demande de rappel était de $33 \%$, et toutes ces demandes ont été honorées ;

- utilisation d'une visioévaluation des dyspnées. Un des enjeux de la régulation médicale des appels concernant la Covid-19 était la possibilité d'évaluer rapidement l'importance de la dyspnée et de l'atteinte respiratoire des patients en sachant que leur interrogatoire téléphonique était biaisé, car la majorité d'entre eux était déjà très anxieuse. Pour cela, la régulation médicale a été dotée d'un système de visio-interrogatoire. Ainsi, l'envoi d'un SMS à l'appelant au cours de l'interrogatoire médical lui permettait de se connecter à un site où son image apparaissait sur l'écran du médecin régulateur. L'état du patient pouvait alors être évalué visuellement avec un protocole d'entretien standardisé. Dans les cas où les éléments précédents ne suffisaient pas, il a été développé au cours de la crise l'envoi d'équipe à domicile avec la possibilité d'une analyse de l'état respiratoire et de l'environnement social du patient. Ces interventions principalement réalisées par des associations de secouristes ou des soignants volontaires ont permis de maintenir à domicile dans de bonnes conditions des patients relevant de la médecine ambulatoire en attendant qu'une visite ou une consultation médicale soit organisée ;

- mise en place d'un contrôle qualité : l'identification des appels concernant la Covid-19 a permis de rappeler à distance les appels pour lesquels l'appelant avait, de lui-même raccroché, sans attendre une réponse ou n'avait pas répondu à un premier rappel après un décroché-raccroché.

L'ensemble des mesures, qui ont été décrites, a été spécifiquement mis en place pour répondre aux appels Covid, certaines mesures avaient été testées ou proposées en amont de la crise. Elles ont constitué un effort d'innovation et d'adaptabilité sans précédent pour la régulation médicale du Samu.

En plus de la modification des organisations de réponse aux appels, les autres missions de la régulation médicale ont été adaptées à la crise et ont aussi bénéficié d'innovations. Ainsi, pour affecter le maximum d'ARM au décroché des appels 15 , la prise des bilans des effecteurs non médicalisés a été réalisée par des personnels paramédicaux, des étudiants, des secouristes encadrés par un médecin régulateur AMU titulaire. Par ailleurs, la recherche de place en réanimation pour les admissions directes des patients pris en charge par le Smur a bénéficié de la création d'une cellule régionale de régulation des lits de réanimation [5]. Cette cellule, mise en place par l'Agence régionale de santé (ARS) et l'AP-HP, a facilité aussi l'organisation des transferts interhospitaliers nécessaires pour optimiser l'utilisation des ressources en réanimation de la région. Le registre opérationnel des ressources (ROR) a été adapté pour permettre de 
visionner en instantané les lits de réanimation disponibles dans les services habituels comme dans les unités ouvertes spécialement pour faire face aux besoins de l'épidémie (dites réa Covid). Cette initiative qui avait été envisagée avant la crise n'avait jamais été réalisée jusque-là.

\section{Renforts en personnels}

Lors du déclenchement de l'épidémie, les effectifs ARM au Samu de Paris sont de 50 équivalents temps plein (ETP). Cet effectif permettait de décrocher au quotidien $95 \%$ des appels 15 dans la minute lorsque le taux d'appels est inférieur à 2500 appels/jour. Dans les crises intenses, mais brèves (attentat terroriste par exemple), le renfort en ressource humaine se traduit le plus souvent par l'augmentation du temps de travail des personnels existants. Entrant dans une crise sanitaire dont l'ampleur était imprévisible, et afin de répondre de façon adaptée à la population, le Samu de Paris, comme tous les autres Samu dans le cadre du plan ORSAN REB, a immédiatement recherché des personnels susceptibles d'apporter une réponse adaptée à la demande en quantité comme en qualité. Cette gestion des renforts en personnel s'est révélée un des points cruciaux de la crise, où l'innovation, l'adaptabilité ont été les plus développées.

Le premier renfort médical est venu des médecins de l'AMU et des psychiatres du service puis des médecins libéraux participant déjà à la PDS (déjà fonctionnelle $24 \mathrm{~h} / 24$ depuis plusieurs années). Le rapprochement avec la médecine libérale a permis non seulement de multiplier par deux puis trois les capacités de régulation de l'activité de soins non programmés, mais aussi d'établir une relation forte avec les structures de consultation (maisons médicales de garde, maisons de santé pluriprofessionnelles, centres de soins...) et avec les dispositifs créés en ville pour répondre spécifiquement à l'épidémie. Des médecins hospitaliers, qui avaient une expérience antérieure de la régulation médicale, ont, dès qu'ils ont été disponibles, rejoint l'équipe de médecins régulateurs. Les internes de médecine d'urgence présents dans le service ont reçu une formation rapide à l'utilisation du logiciel de Centaure 15 et du logiciel CARMEN. Supervisés par les seniors de régulation AMU ou PDS, ils ont rapidement renforcé la régulation médicale en prenant les appels Covid. Les étudiants de DFASM de $3^{\mathrm{e}}$ année ont été affectés à la réception des bilans des secouristes, des ambulances privées ainsi qu'à l'envoi de médecins généralistes.

Devant l'augmentation des appels ainsi que l'efficacité des étudiants de DFASM de $3^{\mathrm{e}}$ année, dès le 6 mars, le doyen de la faculté de médecine Paris-Descartes et la commission de pédagogie ont étendu l'autorisation de recrutement à d'autres étudiants de DFASM de $1^{\mathrm{re}}, 2^{\mathrm{e}}$ et $3^{\mathrm{e}}$ années pour venir renforcer la régulation et assurer les réponses au sein de la salle Covid, dans des fonctions compatibles avec leurs connaissances et leur expérience médicales. Un premier contingent de 70 étudiants en médecine a donc participé dès le début du mois de mars aux réponses aux appels Covid. Ils ont été environ 250 à avoir rejoint le dispositif fin avril. Ces étudiants recevaient une formation à la gestion de l'outil informatique par un membre de l'encadrement du Samu de Paris ainsi qu'un enseignement sur la réalisation d'un interrogatoire ciblé Covid-19. Les modalités d'interrogatoire étaient protocolisées et revues quotidiennement en fonction des recommandations et de l'évolution des connaissances sur l'épidémie. Les étudiants se sont organisés en groupe et ont mutualisé leur apprentissage et leurs acquis. Ils ont assuré notamment, via les réseaux sociaux, la pérennité de leur recrutement. Dans la salle de régulation, ils n'étaient jamais seuls et bénéficiaient, de façon permanente, d'un encadrement de proximité assuré par des médecins AMU et PDS susceptibles de les conseiller, de reprendre à tout moment les appels complexes ou compliqués.

Parallèlement, pour faire face à l'augmentation des interventions des effecteurs et des transports, des renforts d'ambulances de l'Association départementale des transports sanitaires urgents (ATSU) ainsi que des équipes secouristes ont été nécessaires. Pour gérer l'envoi de ces vecteurs et réguler leur destination, un régulateur ambulancier ATSU ainsi que deux opérateurs associatifs ont été déployés $24 \mathrm{~h} /$ 24. Les bilans de ces effecteurs ont été aussi réceptionnés par les étudiants de DFASM de $2^{\mathrm{e}}$ ou $3^{\mathrm{e}}$ années qui, si le bilan était conforme au bilan attendu, pouvaient alors valider la destination prévue, à l'inverse au moindre doute ou incohérence du bilan secouriste devaient en référer à un médecin AMU. L'ensemble de ce dispositif a été maintenu jusqu'au 6 avril puis progressivement diminué.

\section{Rationalisation de la régulation du Samu de Paris}

\section{Sécurisation de la régulation}

Pour éviter la propagation de l'épidémie au sein même de l'équipe du Samu, des mesures de protection et de distanciation sociale ont été mises en place dès le début du mois de mars. Il n'a pas été décidé d'interdire l'accès aux salles de régulation, mais plutôt de le limiter aux personnels en poste et de les responsabiliser au plus vite. Compte tenu de la mutualisation des équipes entre le Smur et le Samu ainsi que la présence de nombreux secouristes, les mesures de désinfection des postes de travail ont été renforcées, le port du masque et l'utilisation de solution hydroalcoolique ont été rendus obligatoires dès le début de l'épidémie. Ces mesures ont été facilement acceptées par les personnels conscients du risque et soucieux de ne pas propager l'épidémie au sein de la 
régulation. Avec la médecine du travail et l'encadrement, les personnes contacts ou paucisymptomatiques ont bénéficié des mêmes traitements et des mêmes précautions que l'ensemble des personnels hospitaliers. La contamination professionnelle est restée très limitée, il n'y a eu aucun cas grave déclaré dans ce contexte.

\section{Optimisation de la réponse au Samu de Paris}

Dans le cadre d'une crise dont la durée et l'intensité n'avaient jusque-là jamais été imaginées, les modifications des pratiques instituées pour y faire face ne pouvaient pas uniquement reposer sur des doctrines ou des intuitions. Adaptabilité, réactivité, et innovation ont été dépendantes de plusieurs facteurs pour qu'elles se transforment en des améliorations concrètes.

\section{Connaissances scientifique et médicale}

La Covid-19 a émergé en France fin janvier 2020. Les informations données par les pays préalablement touchés (en particulier la Chine) étaient intéressantes, mais parcellaires. La réponse aux appelants était conditionnée par les connaissances médicales disponibles à ce moment-là. Un médecin référent a été mis en place : chaque jour, ce médecin du Samu étudiait la littérature publiée en rapport avec la Covid-19, rassemblait les informations et les consignes gouvernementales. Il en faisait la synthèse, qui était diffusée à l'ensemble des intervenants par un bulletin quotidien d'informations permettant une actualisation de la réponse au 15. Des groupes WhatsApp et des conférences téléphoniques ont aussi permis de partager immédiatement les informations et les consignes en cours.

\section{Partage de l'expérience}

Une conférence téléphonique des huit Samu d'Île-de-France a été instituée chaque matin afin d'évaluer l'importance de l'épidémie dans chacun des départements, d'en apprécier l'extension et pour adapter collégialement la réponse. Ces réunions téléphoniques ont permis de recenser et comparer les différentes actions optimisant le décroché du 15 , permettant ainsi une évaluation rapide des mesures et donc son extension ou son arrêt. Ce partage a été aussi possible régulièrement avec les autres Samu de France et sur le plan international avec les services équivalents d'autres pays, notamment européens. Le Samu de Paris a été associé à la conférence téléphonique quotidienne de la cellule de crise AP-HP ainsi qu'à celle organisée par l'ARS Île-de-France, permettant d'avoir une vision globale du dispositif santé de gestion de crise dans lequel l'action du Samu était intégrée.

\section{Analyse en temps réel de l'impact des mesures}

Le soutien du service informatique de l'AP-HP et du groupe INRIA a permis de mettre en place une surveillance renforcée des vitesses de décroché, des temps d'attente des appelants, remplaçant les indicateurs précédents et les tableaux d'affichages qui n'étaient ni fiables ni performants. À partir du 15 mars, une analyse automatisée des indicateurs des quatre Samu AP-HP a été disponible et un écran fiable de supervision des appels a été accessible dans la régulation et au niveau des bureaux de l'encadrement. Cette surveillance a permis à plusieurs reprises une montée en puissance ponctuelle avec l'ensemble des personnels présents dans le Samu en redistribuant les affectations des postes de travail des personnels polyvalents. Elle a aussi guidé l'activation ou la suspension des techniques exposées plus haut (décroché-raccroché, SMS, SVI) et l'affectation des postes informatiques ouverts. Outre l'analyse des différentes phases de la crise, ce bilan quotidien du nombre d'appels comme du temps d'occupation en ligne (TOL), de la quality of service (QoS 60) a permis de confirmer que, dans tous les cas, le nombre d'ARM en poste conditionne le temps de décroché et que la vitesse de décroché réduit non seulement le temps d'attente, mais aussi le nombre d'appels en évitant les appels multiples du même appelant.

\section{Réponses aux appels Covid-19}

Il est trop tôt pour pouvoir évaluer précisément l'impact du rôle d'un ou des Samu dans la crise de la Covid-19. Elle est loin d'être terminée et toute analyse et conclusion hâtives sont discutables. Même si le Samu de Paris a été soumis à une forte tension, notamment vers le 13 mars, l'impact de ce phénomène peut être tempéré par plusieurs points.

L'analyse des pics d'appels a montré qu'il y avait beaucoup de rappels avec des boucles de réentrée. Cette constatation objective un nombre de patients à prendre en charge inférieur au nombre d'appels. L'afflux (et l'attente potentielle) n'était pas du tout continu, mais concentré sur un pic de début de soirée, correspondant à la fin des activités quotidiennes et à la diffusion des informations sur la Covid-19 dans les médias. La majorité des appels portaient sur des patients peu symptomatiques (toux, fièvre modérée) compatibles avec le traitement en ambulatoire. À ce moment-là, aucun report massif des appels sur les autres numéros d'urgence n'a été enregistré au niveau national [6], comme cela se produit sur le 15 quand, par exemple, les autres numéros d'urgences sont sous tension dans un département. Enfin, les patients rappelés après un " décroché-raccroché » étaient satisfaits de ce rappel et des explications fournies. Le dispositif mis en place a permis d'éviter la panique des patients, qui sans régulation et sans contact médical auraient afflué aux SAU. Ils auraient créé des files d'attente dangereuses, car exposant à un surcroît de 
contamination. Ainsi, à Paris, au moment du premier pic d'appels du 13 mars, on peut évaluer à plus de 2000 par jour les patients qui, en l'absence de cette prise en charge téléphonique, se seraient, potentiellement, précipités aux urgences créant des files d'attente, comme on l'a vu dans d'autres pays. Cet afflux aurait aussi accéléré la saturation des hôpitaux avant le deuxième pic d'appels vers le 24 mars qui a porté sur des patients beaucoup plus graves nécessitant des hospitalisations au SAU et en réanimation. Ce deuxième pic a bénéficié d'un dispositif considérablement renforcé aussi bien pour la régulation médicale que pour les interventions préhospitalières. La régulation médicale a donc joué le rôle attendu de « bouclier » de l'hôpital.

L'analyse de cette cinétique a aussi permis de montrer que les appels au 15 étaient un indicateur sensible même s'il n'est pas très spécifique du développement de l'épidémie, et ce paramètre a donc été intégré au tableau de bord de surveillance de la reprise de la circulation du virus $[4,7]$.

\section{Discussion}

Le premier point fort qui s'est dégagé de cette crise est l'adaptabilité du Samu. C'est une illustration de la capacité de résilience dont ont fait preuve les équipes de soins hospitalières et préhospitalières pendant cette épidémie. La modification rapide de l'organisation de la régulation, des procédures, la création de filières de traitement spécifique des appels dédiés à la Covid-19, la collaboration étroite avec la médecine libérale, ainsi que les nombreux renforts en personnels de santé ont permis de faire face à la crise. De même, l'intégration de nouveaux outils de régulation et le déploiement de nouvelles procédures ont permis d'optimiser le décroché du 15 et la réponse médicale à l'ensemble du spectre des appels reçus. Enfin, ces éléments ont nécessité une grande réactivité de la part de l'encadrement et des équipes médicales, paramédicales, administratives, mais aussi techniques, car la connaissance de la maladie, la cinétique de sa propagation et l'évolution de l'activité qui en découlaient se sont modifiées tout au long de la crise. L'épidémie de Covid19 a aussi illustré de manière très concrète l'aspect artificiel de la séparation des urgences médicales en urgences vitales et non vitales. En effet, la cinétique de la maladie et ses présentations cliniques multiples ont montré que la notion de gravité était autant potentielle qu'absolue et nécessitait avant tout une analyse médicale portant certes sur les symptômes, mais aussi sur la temporalité, le terrain, les facteurs de risque et l'environnement, voire l'automédication du patient. Le continuum entre l'urgence ambulatoire relevant de la médecine générale et l'urgence vitale relevant de la réanimation préhospitalière et de l'hospitalisation spécialisée est une réalité. La mise en place du service d'accès aux soins (SAS) présentée dans le pacte de refondation des urgences répond parfaitement à cette question tant sur la plan pratique que sur le plan théorique [8].

Il est aussi indispensable d'envisager des points à améliorer qui prennent tout leur intérêt, en cas de récidive de l'épidémie ou d'une nouvelle crise touchant le domaine de la santé. Au début de la crise de Covid-19, les dotations en matériel et en personnel du Samu de Paris permettaient un fonctionnement opérationnel et la possibilité de faire face à une crise intense, mais brève par la mobilisation immédiate de l'ensemble des personnels. La durée et l'amplitude de la crise ont montré la nécessité d'une mise à niveau des effectifs pour permettre une réactivité immédiate et prolongée. La création d'une réserve de personnel médical, paramédical, d'ARM rapidement mobilisables, et ayant des compétences adaptées à la régulation médicale et au contexte de la crise, est une piste importante d'amélioration. Elle nécessite de disposer d'un programme de développement de formation continue réactualisant les connaissances et mettant à jour documents et protocoles qui doivent pouvoir être très rapidement partagés. Les procédures, les pré-équipements des locaux, les besoins logistiques et matériels n'avaient pas été anticipés pour faire face à une crise si intense sur une si longue durée. Intégrer cette anticipation au dispositif ORSAN REB est une piste d'amélioration très importante. Enfin, disposer d'un SI plus performant, dans lequel toutes les dernières innovations techniques qui ont été utilisées pendant la crise auraient été disponibles de manière native, aurait fait gagner un temps considérable dans l'optimisation de la réponse au 15. D'autres procédures non utilisées pendant la crise pourraient être aussi ajoutées, car elles apporteraient gain de temps et fiabilité dans le recueil des données, par exemple : la transmission automatique du bilan médical incluant l'envoi des informations depuis le terrain vers la régulation médicale, voire vers le service hospitalier receveur. Le déploiement du système d'information national des Samu (SISamu) permettant à tous de disposer des mêmes outils, de partager des informations entre les différents services et d'effectuer des remontées statistiques d'activité est donc un enjeu capital pour affronter dans de meilleures conditions une nouvelle crise. L'épidémie de Covid-19 a aussi démontré la possibilité de mise en tension un numéro d'urgence de façon imprévisible et durable. L'existence de deux numéros d'urgence (l'un secours et l'autre santé) distincts, mais complémentaires est un gage de sécurité, créant aussi une possibilité de suppléance d'un numéro par rapport à l'autre.

S'il est important d'intégrer à nos pratiques les leçons de la crise de Covid-19, il faut cependant ne pas tomber dans l'erreur qui condamnerait à être prisonniers d'un nouveau plan. Ainsi, l'utilisation du plan grippe qui a été développé pour l'épidémie de H1N1 était peu adaptée à l'épidémie de la Covid-19 pour de multiples raisons. La dualité de cette infection pouvant à la fois être bénigne pour certains et extrêmement grave chez d'autres patients et la nécessité du confinement pour bloquer la diffusion du virus en l'absence de 
vaccin sont autant de différences. De même, sur le plan thérapeutique, les moyens de réanimation à mettre en œuvre étaient différents. Pour exemple, l'utilisation de l'assistance circulatoire (ECMO) est restée finalement limitée alors que la durée de ventilation artificielle des patients graves a souvent été très prolongée avec des conséquences sur la disponibilité des respirateurs lourds. De même, si la crise était provoquée par un agent bactérien ou viral plus grave comme le SARS-CoV ou le MERS-CoV ou même comme une fièvre hémorragique de type Ebola, les dispositifs de prise en charge seraient encore différents. Ces remarques soulignent l'importance d'utiliser le dispositif ORSAN et son plan REB comme un outil générique, c'est-à-dire plus comme une boîte à outils que comme une doctrine. Cette constatation avait déjà été faite lors de la prise en charge des attentats terroristes. Elle souligne l'importance sur le terrain de la mise en place de directeurs médicaux de crise (DMC) aptes à comprendre les spécificités d'une maladie complexe et capables de développer des éléments tactiques pour y faire face aussi bien en préhospitalier qu'au sein de l'hôpital. La réalité stratégique du terrain montre l'importance du partage des connaissances et des compétences pour affronter une situation sanitaire exceptionnelle (SSE) comme cela avait été évoqué après les attentats terroristes de 2015 [9].

Parmi les points qui pourraient dans ce nouveau contexte se révéler cruciaux pour gérer la crise, il faut noter que l'épidémie de Covid-19 n'a que peu ou pas touché les enfants. Comme pour les attentats terroristes [10], on ne peut constater que les ressources de soins pédiatriques sont faibles et que la montée en puissance serait beaucoup plus difficile compte tenu de la spécificité de la prise en charge des plus jeunes enfants. L'anticipation d'une telle circonstance ne doit pas être oubliée.

Enfin, dans les points marquants de cette crise, l'information diffusée par les médias a joué un rôle permanent. Dans un contexte où la maladie est peu connue, les multiplications d'avis d'experts parfois contradictoires ont certainement influencé le comportement de la population. Il serait important d'analyser spécifiquement cet aspect qui a pu conduire dans certains cas à une autocensure des soins concernant aussi bien l'épidémie en cours que des maladies chroniques. Dans le cadre de la récidive de la crise ou d'une autre qui pourrait être plus intense ou plus longue, le rôle de la communication pourrait être amélioré et devenir un véritable atout pour favoriser et maintenir la continuité des soins courants tout en traitant une SSE.

\section{Conclusion}

L'analyse de l'expérience acquise au cours de la crise de Covid-19 au Samu de Paris a permis de dégager des éléments remarquables comme des pistes d'amélioration dont la pertinence peut être généralisée à d'autres Samu. Elle a porté sur l'adaptabilité et la coopération entre l'ensemble des Samu. L'enseignement d'une capacité de médecine de catastrophe, dispensé principalement aux médecins de Samu depuis de nombreuses années, a facilité la maîtrise des grands principes de réaction face à une SSE. La réalisation en IDF d'exercices annuels impliquant les différents Samu d'IDF a facilité leur coopération. La possibilité d'adaptation des moyens à l'activité a été essentielle et possible grâce à des recrutements instantanés et à une formation rapide de personnels. Le stress test de la Covid-19 a donc démontré que la mise à niveau des effectifs du quotidien était un préalable indispensable à une extension rapide et efficace de la réponse face à un événement exceptionnel d'intensité majeure.

Liens d'intérêts : les auteurs déclarent ne pas avoir de liens d'intérêts. 


\section{Éléments remarquables}

- Modification rapide des procédures de régulation avec identification d'une filière d'appels Covid-19 Intensification de la collaboration avec la médecine libérale

- Création et déploiement de ressources en personnels dédiés Covid

- Réaffectation des personnels disponibles et recrutement de renfort en fonction de l'évolution de l'épidémie

- Mise en place très rapide de formations spécifiques Covid-19 pour les titulaires et les renforts : médecins étudiants, autres professionnels de santé...

- Formation des médecins urgentistes à la médecine de catastrophe - Intégration de nouveaux outils : visioconsultation, message type SMS

- Déploiement de nouvelles procédures : décroché-raccroché, gestion des appels avec niveaux de réponse de niveaux 1 et 2 (N1 et N2)

- Adaptation journalière des pratiques à l'évolution de l'épidémie

à la connaissance du virus

- Communication à tous les niveaux entre professionnels

et administration par des outils numériques

- Suivi de l'activité et mise en place de paramètres d'évaluation en continu pendant la crise

- Création et mise en service d'un tableau de bord hospitalier

incorporant le nombre d'appels au Samu

\section{Points d'amélioration}

- Mise à niveau préalable à toute crise des effectifs en assistants de régulation médicale (ARM) et en médecins

- Création d'une réserve de personnel et de compétence, rapidement mobilisable, ayant déjà pratiqué la régulation médicale comme médecin ou comme ARM - Développement d'une formation continue avec réactualisation des connaissances en prévision d'une reprise de l'épidémie

- Création et actualisation de documents et protocoles destinés aux personnels complémentaires

- Dotation en matériel pour les besoins du quotidien correspondant au développement des nouvelles technologies (téléconsultations, connexion et interfaçage des logiciels) - Dotation en matériel permettant l'activation rapide des salles de régulation complémentaires

- Pré-équipements des locaux de régulation complémentaires - Anticipation des besoins logistiques et matériels pour une crise de longue durée (protection, désinfection, maintenance) - Procédures d'activation des salles de régulation complémentaires sur des indicateurs de qualité

\section{Références}

1. Ministère des Solidarités et de la Santé (2019) Plan ORSAN REB. In: Guide d'aide à la préparation et à la gestion des tensions hospitalières et des situations sanitaires exceptionnelles. https://solidarites-sante.gouv.fr/systeme-de-sante-et-medico-social/securite-sanitaire/guide-gestion-tensions-hospitalieres-SSE. https://solidaritessante.gouv.fr/IMG/pdf/guide_situation_sanitaire_exceptionnelle. pdf (Dernier accès le 15 juillet 2020)

2. Ministère des Solidarités et de la Santé (2019) Préparation au risque épidémique Covid-19, établissements de santé, médecine de ville établissements médico-sociaux ; Guide méthodologique édition 20 février 2019 : https://solidarites-sante.gouv.fr/IMG/ pdf/guide methodologique Covid-19-2.pdf (Dernier accès le 15 juillet 2020 )

3. Lamhaut L, Nivet MC, Dagron C, et al (2020) Retour d'expérience des évacuations par train à grande vitesse de patients en syndrome de détresse respiratoire aiguë sur infection à Covid19 : les missions CHARDON. Ann Fr Med Urg 10:288-97

4. Gaubert S, Akian M, Allamigeon X, et al (2020) Understanding and monitoring the evolution of the Covid-19 epidemic from medical emergency calls: the example of the Paris. C R Math Acad Sci (Paris) 358 (in press)

5. Collaboration AP-HP/Universités/Inserm Covid-19 (2020) Retour d'expérience sur COVIDOM : une solution de télésurveillance pour les patients porteurs ou suspectés Covid-19. Ann Fr Med Urg 10:314-20

6. Braun F (2020) Une crise sanitaire avant tout. Ann Fr Med Urg 10:199-201

7. Piarroux R, Batteux F, Rebaudet S, Boëlle PY (2020) Les indicateurs d'alerte et de surveillance de la Covid-19. Ann Fr Med Urg 10:333-7

8. Mesnier T, Carli P (2019) Rapport : Pour un pacte de refondation des urgences. https: solidarités-sante.gouv.fr/IMG/pdf/rapport_pour_un_pacte_de_refondation_des_urgences_2019-058r.pdf (Dernier accès le 15 juillet 2020)

9. Braun F, Carli P (2017) Coordonnateur des opérations médicales : le DSM 2.0. JEUR 29:208-11

10. Carli P, Pons F, Levraut J, et al (2017) The French emergency medical services after the Paris and Nice terrorist attacks: what have we learnt? Lancet 390:2735-8 